\title{
Some fixed point results in fuzzy cone normed linear space
}

\author{
Phurba Tamang ${ }^{1 *}$ (D) and Tarapada Bag ${ }^{2}$
}

\author{
${ }^{*}$ Correspondence: \\ phurbat254@gmail.com \\ 'Department of Mathematics, Surya \\ Sen Mahavidyalaya, Siliguri 734004, \\ India \\ Full list of author information is \\ available at the end of the article
}

\begin{abstract}
In this paper, the well known fixed point theorems of Banach, Kannan, and Chatterjee are extended to the fuzzy cone normed linear space.
\end{abstract}

Keywords: Fuzzy cone norm, Strongly minihedral cone, $\alpha$-convergent, $\alpha$-Cauchy Mathematics subject classification: 46S40, 03E72, 32C25

\section{Introduction}

The concept of fuzzy norm was first introduced by Katsaras [1] in the year 1984. After that, in 1992, Felbin [2] defined a fuzzy norm on a linear space with an associated metric of the Kaleva and Seikkala type [3]. Further developement in the notion of fuzzy norm took place in 1994, when Cheng and Moderson [4] gave the definition of fuzzy norm in another approach having an associated metric of the Kramosil and Michalek type [5] . Thereafter, following the definition of fuzzy norm by Cheng and Moderson [4], Bag and Samanta [6] introduced the concept of fuzzy norm in a different way.

On the other hand, several authors generalized the concept of metric space in many ways. One of them is the notion of cone metric space introduced by Long-Guang et al. [7] in the year 2007. In the year 2017, Tamang and Bag [8] extended the concept of fuzzy norm to fuzzy cone norm with replacement of $\mathrm{R}$ by a real Banach space. In 1922, Banach [9] proved fixed point result on contractive type mappings. So far, many authors have obtained interesting extensions and generalization of the Banach contraction principle. In 1968, Kannan [10] and, in 1972, Chatterjee [11] studied contractive mappings which gives unique fixed point on complete metric space. As the fuzzy mathematics along with the classical ones are constantly developing, the above fixed point results in fuzzy cone normed linear space setting can also play an important role. Our aim in this paper is to establish Banach, Kannan, and Chatterjee type fixed point theorems in fuzzy cone normed linear space setting.

\section{Preliminaries}

In this section, some essential concepts for study are stated. Throughout the paper we use symbol $\bigwedge$ to denote the infimum.

Definition 1 [7] Let E be a real Banach space and P be a subset of E. P is called a cone if and only if:

(c) The Author(s). 2019 Open Access This article is distributed under the terms of the Creative Commons Attribution 4.0 International License (http://creativecommons.org/licenses/by/4.0/), which permits unrestricted use, distribution, and reproduction in any medium, provided you give appropriate credit to the original author(s) and the source, provide a link to the Creative Commons license, and indicate if changes were made. 
(i) $P$ is closed, nonempty and $P \neq\{\theta\}$;

(ii) $a, b \in R, a, b \geq 0, x, y \in P \Rightarrow a x+b y \in P$;

(iii) $x \in P$ and $-x \in P \Rightarrow x=\theta$.

Given a cone $P \subset E$, we define a partial ordering $\preceq$ with respect to $P$ by $x \preceq y$ iff $y-x \in P$. We shall write $x \prec y$ to indicate that $x \preceq y$ but $x \neq y$ while $x<<y$ will stand for $y-x \in \operatorname{Int} P$, where $\operatorname{Int} P$ denotes the interior of $P$.

The cone $P$ is called normal if there is a number $K>0$ such that for all $x, y \in E$, with $\theta \preceq x \preceq y$ implies $\|x\| \leq K\|y\|$.

The least positive number satisfying above is called the normal constant of $P$.

The cone $P$ is called regular if every increasing sequence which is bounded from above is convergent. That is if $\left\{x_{n}\right\}$ is a sequence in $E$ such that

$$
x_{1} \preceq x_{2} \preceq \cdots \preceq x_{n} \preceq \cdots \preceq y
$$

for some $y \in E$, then there is $x \in E$ such that $\left\|x_{n}-x\right\| \rightarrow 0$ as $n \rightarrow \infty$.

Equivalently, the cone $P$ is regular if every decreasing sequence is bounded below is convergent. It is clear that a regular cone is a normal cone.

Definition 2 [12] The cone $P$ is called strongly minihedral if every subset of $E$ which is bounded above via the partial ordering obtained by $P$, must have a least upper bound. Hence, every subset which is bounded below must have greatest lower bound.

Definition 3 [13] A binary operation $*:[0,1] \times[0,1] \rightarrow[0,1]$ is a t-norm if it satisfies the following conditions:

(1) $*$ is associative and commutative;

(2) $a * 1=a \quad \forall a \in[0,1]$

(3) $a * b \leq c * d$ whenever $a \leq c$ and $b \leq d$ for each $a, b, c, d \in[0,1]$.

If $*$ is continuous then it is called continuous t-norm. The following are examples of some t-norms that are frequently used and defined for all $a, b \in[0,1]$.

(i) Standard intersection: $a * b=\min (a, b)$.

(ii) Algebraic product: $a * b=a b$.

(iii) Bounded difference: $a * b=\max (0, a+b-1)$.

(iv) Drastic intersection:

$$
a * b=\left\{\begin{array}{l}
a \text { for } b=1 \\
b \text { for } a=1 \\
0 \text { otherwise }
\end{array}\right.
$$

Definition 4 [8] Let $X$ be a linear space over the field $K$ and $E$ be a real Banach space with cone $P, *$ is a t-norm. A fuzzy subset $N_{c}: X \times E \longrightarrow[0,1]$ is said to be a fuzzy cone norm if

(FCN1) $\forall t \in E$ with $t \preceq \theta_{E}, N_{c}(x, t)=0$;

$(F C N 2) \quad\left(\forall \theta_{E} \prec t, N_{c}(x, t)=1\right)$ iff $x=\theta_{X}$;

(FCN3) $\forall \theta_{E} \prec t$ and $0 \neq c \in K, N_{c}(c x, t)=N_{c}\left(x, \frac{t}{|c|}\right)$;

(FCN4) $\forall x, y \in X$ and $s, t \in E, N_{c}(x+y, s+t) \geq N_{c}(x, s) * N_{c}(y, t)$;

(FCN5) $\lim _{\|t\| \rightarrow \infty} N_{c}(x, t)=1$;

Then $\left(X, N_{c}, *\right)$ is said to be a fuzzy cone normed linear space w.r.t $E$. 
Theorem 1 (Banach [9]) Let f be a self-map of a complete metric space $(X, d)$ such that $d(f(x), f(y)) \leq \alpha d(x, y)$ for some real number $\alpha, 0<\alpha<1$ for each $x, y \in X$. Then $f$ has $a$ unique fixed point.

Theorem 2 (Kannan [10]) Letfbe a self-map of a complete metric space $(X, d)$ such that $d(f(x), f(y)) \leq \beta[d(f(x), x)+d(f(y), y)]$ for some real number $\beta, 0<\beta<\frac{1}{2}$ for each $x, y \in X$. Then $f$ has a unique fixed point.

Theorem 3 (Chatterjee [11]) Let $f$ be a self-map of a complete metric space $(X, d)$ such that $d(f(x), f(y)) \leq \gamma[d(f(x), y)+d(f(y), x)]$ for some real number $\gamma, 0<\gamma<\frac{1}{2}$ for each $x, y \in X$. Then $f$ has a unique fixed point.

\section{Main results}

In this section we modified Definition 4 of fuzzy cone normed linear space as follows in order to develop some fixed point results.

Definition 5 Let $X$ be a linear space over the field $K$ and $E$ be a real Banach space with cone $P, *$ is a $t$-norm. A fuzzy subset $N_{c}: X \times E \longrightarrow[0,1]$ is said to be a fuzzy cone norm if

(FCN1) $\forall t \in E$ with $t \preceq \theta_{E}, N_{c}(x, t)=0$;

$(F C N 2)\left(\forall \theta_{E} \prec t, N_{c}(x, t)=1\right)$ iff $x=\theta_{X}$;

$\left(\theta_{X}\right.$ denotes the zero element of $\left.X\right)$

(FCN3) $\forall \theta_{E} \prec t$ and $0 \neq c \in K, N_{c}(c x, t)=N_{c}\left(x, \frac{t}{|c|}\right)$;

(FCN4) $\forall x, y \in X$ and $s, t \in E, N_{c}(x+y, s+t) \geq N_{c}(x, s) * N_{c}(y, t)$;

(FCN5) $N_{c}(x, t)=1$ if $s \prec t \forall s \in P$;

Then $\left(X, N_{c}, *\right)$ is said to be a fuzzy cone normed linear space w.r.t. $E$.

(FCN6) $N_{c}(x, t)>0 \forall t \succ \theta_{E} \Rightarrow x=\theta_{X}$.

Note. We notice that for a real Banach space with normal cone the modified definition is stronger than the existing one.

For, $s \prec t \forall s \in P$

i.e, $\theta_{E} \preceq s \prec t \forall s \in P$

$\Rightarrow\|s\| \leq K\|t\| \forall s \in P(K$ is a normal constant $)$

$\Rightarrow\|t\|>$ any positive real number

$\Rightarrow\|t\| \longrightarrow \infty$.

Definition 6 Let $\left(X, N_{c}, *\right)$ be a fuzzy cone normed linear space with a strongly minihedral cone $P$ and $\alpha \in(0,1)$. A sequence $\left\{x_{n}\right\}$ is said to be $\alpha$-fuzzy convergent and converges to $x$ if $\lim _{n \rightarrow \infty} \bigwedge\left\{t>\theta_{E}: N_{c}\left(x_{n}-x, t\right) \geq \alpha\right\}=\theta_{E}$. If $\lim _{n \rightarrow \infty} \bigwedge\left\{t>\theta_{E}: N_{c}\left(x_{n}-x, t\right) \geq \alpha\right\}=$ $\theta_{E} \quad \forall \alpha \in(0,1)$, then $\left\{x_{n}\right\}$ is said to be l-fuzzy convergent and converges to $x$.

Definition 7 Let $\left(X, N_{c}, *\right)$ be a fuzzy cone normed linear space with a strongly minihedral cone $P$ and $\alpha \in(0,1)$. A sequence $\left\{x_{n}\right\}$ is said to be $\alpha$-fuzzy Cauchy sequence if $\lim _{n \rightarrow \infty} \bigwedge\left\{t \succ \theta_{E}: N_{c}\left(x_{n+p}-x_{n}, t\right) \geq \alpha\right\}=\theta_{E}$. for each $p=1,2,3, \ldots$ If $\lim _{n \rightarrow \infty} \bigwedge\left\{t \succ \theta_{E}: N_{c}\left(x_{n+p}-x_{n}, t\right) \geq \alpha\right\}=\theta_{E} \quad \forall \alpha \in(0,1)$ and for each $p=1,2,3, \ldots$, then $\left\{x_{n}\right\}$ is said to be l-fuzzy Cauchy sequence. 
Definition 8 Let $\left(X, N_{c}, *\right)$ be a fuzzy cone normed linear space with a strongly minihedral cone $P$ and $\alpha \in(0,1)$. Then, $X$ is said to be $\alpha$-fuzzy complete if every $\alpha$-fuzzy Cauchy sequence is $\alpha$-fuzzy convergent to some element in $X$.

Definition 9 Let $\left(X, N_{c}, *\right)$ be a fuzzy cone normed linear space with a strongly minihedral cone $P$ and $\alpha \in(0,1)$. Then $X$ is said to be l-fuzzy complete if every $\alpha$-fuzzy Cauchy sequence is $\alpha$-fuzzy convergent $\forall \alpha \in(0,1)$.

Example 1 Let $\left(X,\|\|_{c}\right)$ be a cone normed linear space and take $E=R^{2}$. Then $P=$ $\left\{\left(t_{1}, t_{2}\right): t_{1}, t_{2} \geq 0\right\} \subset E$ is a strongly minihedral normal cone with normal constant 1. Define a function $N_{c}: X \times E \longrightarrow[0,1]$ by

$$
\begin{aligned}
N_{c}(x, t)=1 & \text { if }\|x\|_{c} \prec t \\
=0 & \text { if } t \preceq\|x\|_{c}
\end{aligned}
$$

If we choose $*=\min$, Then $\left(X, N_{c}, *\right)$ is a fuzzy cone normed linear space satisfying (FCN6). If we take $X=R$, then $\left(X, N_{c}, *\right)$ is an $l$-fuzzy complete fuzzy cone normed linear space

Proof:

(i) $\forall t \in E$ with $t \preceq \theta_{E}$, we have by definition, $N_{c}(x, t)=0$ for all $x \in X$. Thus (FCN1) holds.

(ii) $\forall t \in E$ with $\theta_{E} \prec t$,

$N_{c}(x, t)=1$

$\Rightarrow\|x\|_{c} \prec t \forall t \succ \theta_{E}$

$\Rightarrow\|\| x\left\|_{c}\right\| \leq\|t\| \forall t \succ \theta_{E}$ (since $P$ is normal cone with normal constant 1)

$\Rightarrow\|\| x\left\|_{c}\right\|=0$.

$\Rightarrow\|x\|_{c}=\theta_{E}$.

$\Rightarrow x=\theta_{X} \quad\left(\theta_{X}\right.$ denotes the zero element of $\left.X\right)$

Again $x=\theta_{X}$

$\Rightarrow\|x\|_{c}=\theta_{E}$.

$\Rightarrow\left\|\theta_{X}\right\|_{c} \prec t \forall t \succ \theta_{E}$

$\Rightarrow N_{c}(x, t)=1$.

So (FCN2) holds.

(iii) For all $t \in E$ with $\theta_{E} \prec t$ and $0 \neq c \in K$

Let $N_{c}(c x, t)=0$

$\Rightarrow t \preceq\|c x\|_{c}$

$\Rightarrow t \preceq|c|\|x\|_{c}$

$\Rightarrow \frac{t}{|c|} \preceq\|x\|_{c} \Rightarrow N_{c}\left(x, \frac{t}{|c|}\right)=0$.

Let $N_{c}(c x, t)=1$

$\Rightarrow\|c x\|_{c} \preceq t$

$\Rightarrow|c|\|x\|_{c} \preceq t$

$\Rightarrow\|x\|_{c} \preceq \frac{t}{|c|}$

$\Rightarrow N_{c}\left(x, \frac{t}{|c|}\right)=1$.

So (FCN3) holds.

(iv) We have to show that

$N_{c}(x+u, s+t) \geq \min \left\{N_{c}(x, s), N_{c}(u, t)\right\} \quad \forall x, y \in X$ and $s, t \in E$ 
If $N_{c}(x+u, s+t)=0$

Then, $s+t \preceq\|x+u\|_{c} \preceq\|x\|_{c}+\|u\|_{c}$

$$
\begin{aligned}
& \Rightarrow\|x\|_{c}+\|u\|_{c}-(s+t) \in P \\
& \Rightarrow\|u\|_{c}-t-\left(s-\|x\|_{c}\right) \in P \\
& \Rightarrow s-\|x\|_{c} \preceq\|u\|_{c}-t
\end{aligned}
$$

If $\|x\|_{c} \prec s$ i.e, $\theta_{E} \prec s-\|x\|_{c}$, then from (1)

$\theta_{E} \prec\|u\|_{c}-t$

$\Rightarrow t \prec\|u\|_{c}$

So if $\|x\|_{c} \prec s$, then $t \prec\|u\|_{c}$

So $N_{c}(x, s)=1$ and $N_{c}(u, t)=0$.

Similarly, if $\|u\|_{c} \prec t$, then $s \prec\|x\|_{c}$

So $N_{c}(u, t)=1$ and $N_{c}(x, s)=0$.

So in both cases,

$N_{c}(x+u, s+t) \geq \min \left\{N_{c}(x, s), N_{c}(u, t)\right\}=0$.

If $N_{c}(x+u, s+t)=1$

Then $N_{c}(x+u, s+t) \geq \min \left\{N_{c}(x, s), N_{c}(u, t)\right\}$

So (FCN4) holds.

(v) If $s \prec t$ for every $s \in P$, then by definition, $N_{c}(x, t)=1$. So (FCN5) holds.

Thus, $\left(X, N_{c}, *\right)$ is a fuzzy cone normed linear space.

Now, $\forall t>\theta_{E}, N_{c}(x, t)>0$

$\Rightarrow N_{c}(x, t)=1, \forall t \succ \theta_{E}$

$\Rightarrow\|x\|_{c} \prec t \forall t \succ \theta_{E}$

$\Rightarrow\|\| x\left\|_{c}\right\| \leq\|t\| \forall t \succ \theta_{E} \quad$ (P is a normal cone with normal constant 1)

$\Rightarrow\|x\|_{c}=\theta_{E}$

$\Rightarrow x=\theta_{X}$.

Thus, (FCN6) holds.

We now prove that $\left(X, N_{c}, *\right)$ is an 1 -fuzzy complete cone normed linear space.

Let $\left\{x_{n}\right\}$ be a $\alpha$-Cauchy sequence in $\left(X, N_{c}, *\right)$ for $\alpha \in(0,1)$.

Then $\bigwedge\left\{t \succ \theta_{E}: N_{c}\left(x_{n}-x_{m}, t\right) \geq \alpha\right\}=\theta_{E}$ as $m, n \rightarrow \infty$

Choose $\epsilon \succ \theta_{E}$ arbitrarily, then there exists a natural number $p$ such that

$\bigwedge\left\{t \succ \theta_{E}: N_{c}\left(x_{n}-x_{m}, t\right) \geq \alpha\right\} \prec \epsilon \forall t \succ \theta_{E}$ and $m, n \geq p$.

$\Rightarrow N_{c}\left(x_{n}-x_{m}, \epsilon\right) \geq \alpha>0 \forall \epsilon \succ \theta_{E}$ and $m, n \geq p$.

$\Rightarrow\left\|x_{n}-x_{m}\right\|_{c} \prec \epsilon \forall \epsilon \succ \theta_{E}$ and $m, n \geq p$. (by the definition of $N_{c}$ )

$\Rightarrow\|\| x_{n}-x_{m}\left\|_{c}\right\| \leq\|\epsilon\| \forall \epsilon \succ \theta_{E}$ (since $P$ is normal cone with normal constant 1)

$\Rightarrow\left\|x_{n}-x_{m}\right\|_{c} \rightarrow \theta_{E}$ as $m, n \rightarrow \infty$

$\Rightarrow\left|x_{n}-x_{m}\right| \rightarrow 0$ as $m, n \rightarrow \infty$

$\Rightarrow\left\{x_{n}\right\}$ is a Cauchy sequence in $R$. Since $R$ is complete, $\exists x \in R$ such that $x_{n} \rightarrow x$ as

$n \rightarrow \infty$

$\Rightarrow x_{n}-x \rightarrow 0$ as $n \rightarrow \infty$

$\Rightarrow\left\|x_{n}-x\right\|_{c} \rightarrow \theta_{E}$ as $n \rightarrow \infty$

$\Rightarrow$ there exists a natural number $n_{0}(t)$ such that $\left\|x_{n}-x\right\|_{c} \prec t \forall t \succ \theta_{E}$ and $n \geq n_{0}(t)$.

$\Rightarrow N_{c}\left(x_{n}-x, t\right)=1 \forall t \succ \theta_{E}$ and $n \geq n_{0}(t)$.

$\Rightarrow \bigwedge\left\{t \succ \theta_{E}: N_{c}\left(x_{n}-x, t\right) \geq \alpha\right\}=\theta_{E}$ as $n \rightarrow \infty$

$\Rightarrow\left\{x_{n}\right\}$ is $\alpha$-convergent to $x$. 
Since $\alpha \in(0,1)$ is arbitrary, every $\alpha$-Cauchy sequence is $\alpha$-convergent. So $\left(X, N_{c}, *\right)$ is an 1 -fuzzy complete fuzzy cone normed linear space.

\section{Some fixed point theorems}

In this section Banach, Kannan, and Chatterjee type fixed point theorems are established in fuzzy setting.

Throughout this section, we consider $*$ as continuous t-norm.

Theorem 4 (Banach Contraction type theorem in fuzzy cone normed linear space)

Let $\left(X, N_{c}, *\right)$ be an $l$-fuzzy complete cone normed linear space satisfying $(F C N 6), P$ be a stronghly minihedral cone with normal constant $M$. Suppose the mapping $T: X \longrightarrow X$ satisfies the contractive condition

$$
\bigwedge\left\{t>\theta_{E}: N_{c}(T x-T y, t) \geq \alpha\right\} \preceq k \bigwedge\left\{t>\theta_{E}: N_{c}(x-y, t) \geq \alpha\right\}
$$

for some, $\alpha \in(0,1)$ and $k \in(0,1)$ is a constant. Then, $T$ has a fixed point in $X$. In addition if $M=1$, then the fixed point is unique. Assuming that $\beta * \beta>0 \forall \beta \in(0,1)$.

Proof Choose $x_{0} \in X$. Set $x_{1}=T x_{0}, x_{2}=T x_{1}=T^{2} x_{0}, \ldots, x_{n+1}=T x_{n}=T^{n+1} x_{0}$. So $\left\{x_{n}\right\}$ is a sequence in $X$. First, we show that $\left\{x_{n}\right\}$ is $\beta$-Cauchy sequence for some $\beta \in(0,1)$.

Now, for some $\alpha \in(0,1)$;

$$
\begin{aligned}
& \begin{aligned}
\left.\bigwedge t \succ \theta_{E}: N_{c}\left(x_{n+1}-x_{n}, t\right) \geq \alpha\right\} & =\bigwedge\left\{t>\theta_{E}: N_{c}\left(T x_{n}-T x_{n-1}, t\right) \geq \alpha\right\} \\
& \leq k \bigwedge\left\{t \succ \theta_{E}: N_{c}\left(x_{n}-x_{n-1}, t\right) \geq \alpha\right\}
\end{aligned} \\
& \text { i.e., } \bigwedge\left\{t \succ \theta_{E}: N_{c}\left(x_{n+1}-x_{n}, t\right) \geq \alpha\right\} \preceq k \bigwedge\left\{t>\theta_{E}: N_{c}\left(x_{n}-x_{n-1}, t\right) \geq \alpha\right\}
\end{aligned}
$$

Proceeding similarly, we have

$$
\bigwedge\left\{t>\theta_{E}: N_{c}\left(x_{n+1}-x_{n}, t\right) \geq \alpha\right\} \preceq k^{n} \bigwedge\left\{t>\theta_{E}: N_{c}\left(x_{1}-x_{0}, t\right) \geq \alpha\right\}
$$

Using normality condition, we get

$$
\begin{aligned}
& \left\|\bigwedge\left\{t \succ \theta_{E}: N_{c}\left(x_{n+1}-x_{n}, t\right) \geq \alpha\right\}\right\| \leq k^{n} M\left\|\bigwedge\left\{t \succ \theta_{E}: N_{c}\left(x_{1}-x_{0}, t\right) \geq \alpha\right\}\right\| \\
& \Rightarrow \lim _{n \rightarrow \infty}\left\|\bigwedge\left\{t \succ \theta_{E}: N_{c}\left(x_{n+1}-x_{n}, t\right) \geq \alpha\right\}\right\|=0\left(\text { since } k^{n} \rightarrow 0 \text { as } n \rightarrow \infty\right) \\
& \Rightarrow \lim _{n \rightarrow \infty} \bigwedge\left\{t \succ \theta_{E}: N_{c}\left(x_{n+1}-x_{n}, t\right) \geq \alpha\right\}=\theta_{E}
\end{aligned}
$$

Now for $p \geq 1$, we have

$$
\begin{aligned}
& \bigwedge\left\{t \succ \theta_{E}: N_{c}\left(x_{n+p}-x_{n+p-1}, \frac{t}{p}\right) \geq \alpha\right\} \\
& +\bigwedge\left\{t \succ \theta_{E}: N_{c}\left(x_{n+p-1}-x_{n+p-2}, \frac{t}{p}\right) \geq \alpha\right\} \\
& +\ldots+\bigwedge\left\{t \succ \theta_{E}: N_{c}\left(x_{n+1}-x_{n}, \frac{t}{p}\right) \geq \alpha\right\} \\
& \geq \bigwedge\left\{t \succ \theta_{E}: N_{c}\left(x_{n+p}-x_{n}, t\right) \geq \alpha * \alpha * \ldots * \alpha\right\}
\end{aligned}
$$

Since $*$ is continuous, $\exists \beta \in(0,1)$ such that $\alpha * \alpha * \ldots * \alpha=\beta$. 
From above, we get

$$
\begin{aligned}
& p \bigwedge\left\{t \succ \theta_{E}: N_{c}\left(x_{n+p}-x_{n+p-1}, t\right) \geq \alpha\right\}+p \bigwedge\left\{t \succ \theta_{E}: N_{c}\left(x_{n+p-1}-x_{n+p-2}, t\right) \geq \alpha\right\} \\
& +\ldots+p \bigwedge\left\{t \succ \theta_{E}: N_{c}\left(x_{n+1}-x_{n}, t\right) \geq \alpha\right\} \succeq \bigwedge\left\{t \succ \theta_{E}: N_{c}\left(x_{n+p}-x_{n}, t\right) \geq \beta\right\}
\end{aligned}
$$

Using normality condition, we have,

$$
\begin{aligned}
& \left\|\bigwedge\left\{t \succ \theta_{E}: N_{c}\left(x_{n+p}-x_{n}, t\right) \geq \beta\right\}\right\| \leq p M\left\|\bigwedge\left\{t \succ \theta_{E}: N_{c}\left(x_{n+p}-x_{n+p-1}, t\right) \geq \alpha\right\}\right\| \\
+ & p M\left\|\bigwedge\left\{t \succ \theta_{E}: N_{c}\left(x_{n+p-1}-x_{n+p-2}, t\right) \geq \alpha\right\}\right\|+\ldots \\
+ & p M\left\|\bigwedge\left\{t \succ \theta_{E}: N_{c}\left(x_{n+1}-x_{n}, t\right) \geq \alpha\right\}\right\| \\
\Rightarrow & \left\|\bigwedge\left\{t \succ \theta_{E}: N_{c}\left(x_{n+p}-x_{n}, t\right) \geq \beta\right\}\right\| \rightarrow 0 \text { as } n \rightarrow \infty \text { for } p=1,2,3, \ldots \text { using }(2) \\
\Rightarrow & \bigwedge\left\{t \succ \theta_{E}: N_{c}\left(x_{n+p}-x_{n}, t\right) \geq \beta\right\} \rightarrow \theta_{E} \text { as } n \rightarrow \infty \text { for } p=1,2,3, \ldots \\
\Rightarrow & \left\{x_{n}\right\} \text { is a } \beta \text {-Cauchy sequence. } \\
& \text { So } \exists x \in X \text { such that } \lim _{n \rightarrow \infty} \bigwedge\left\{t \succ \theta_{E}: N_{c}\left(x_{n}-x, t\right) \geq \beta\right\}=\theta_{E} .
\end{aligned}
$$

We have

$$
\begin{aligned}
& \bigwedge\left\{s+t \succ \theta_{E}: N_{c}(T x-x, s+t) \geq \beta * \beta\right\} \\
& \preceq \bigwedge\left\{s \succ \theta_{E}: N_{c}\left(T x-x_{n}, s\right) \geq \beta\right\}+\bigwedge\left\{t \succ \theta_{E}: N_{c}\left(x_{n}-x, t\right) \geq \beta\right\} \\
& \preceq k \bigwedge\left\{s \succ \theta_{E}: N_{c}\left(x-x_{n-1}, s\right) \geq \beta\right\}+\bigwedge\left\{t \succ \theta_{E}: N_{c}\left(x_{n}-x, t\right) \geq \beta\right\}
\end{aligned}
$$

Using normality condition and (3), we get

$$
\begin{aligned}
& \left\|\bigwedge\left\{s+t \succ \theta_{E}: N_{c}(T x-x, s+t) \geq \beta * \beta\right\}\right\| \\
& \leq M\left\|k \bigwedge\left\{s \succ \theta_{E}: N_{c}\left(x-x_{n-1}, s\right) \geq \beta\right\}+\bigwedge\left\{t \succ \theta_{E}: N_{c}\left(x_{n}-x, t\right) \geq \beta\right\}\right\| \\
& \leq M k\left\|\bigwedge\left\{s \succ \theta_{E}: N_{c}\left(x-x_{n-1}, s\right) \geq \beta\right\}\right\|+M\left\|\bigwedge\left\{t \succ \theta_{E}: N_{c}\left(x_{n}-x, t\right) \geq \beta\right\}\right\|
\end{aligned}
$$

Taking limit as $n \rightarrow \infty$, we have

$$
\begin{aligned}
& \left\|\bigwedge\left\{s+t \succ \theta_{E}: N_{c}(T x-x, s+t) \geq \beta * \beta\right\}\right\|=0 \\
& \Rightarrow \bigwedge\left\{s+t \succ \theta_{E}: N_{c}(T x-x, s+t) \geq \beta * \beta\right\}=\theta_{E} \\
& \Rightarrow \forall(s+t) \succ \theta_{E} ; N_{c}(T x-x, s+t) \geq \beta * \beta>0 \\
& \Rightarrow T x-x=\theta_{X} \text { by }(\text { FCN6) } \\
& \Rightarrow T x=x .
\end{aligned}
$$

Thus, $x$ is a fixed point of $T$.

Uniqueness: If possible suppose that $\exists y \in X$ such that $T y=y$.

$$
\text { Now, } \begin{aligned}
\bigwedge\left\{t \succ \theta_{E}: N_{c}(x-y, t) \geq \alpha\right\} & =\bigwedge\left\{t \succ \theta_{E}: N_{c}(T x-T y, t) \geq \alpha\right\} \\
& \preceq k \bigwedge\left\{t \succ \theta_{E}: N_{c}(x-y, t) \geq \alpha\right\}
\end{aligned}
$$

Using normality condition with normal constant 1 , we have 


$$
\begin{aligned}
& \left\|\bigwedge\left\{t \succ \theta_{E}: N_{c}(x-y, t) \geq \alpha\right\}\right\| \leq k\left\|\bigwedge\left\{t \succ \theta_{E}: N_{c}(x-y, t) \geq \alpha\right\}\right\| \\
& \Rightarrow(1-k)\left\|\bigwedge\left\{t \succ \theta_{E}: N_{c}(x-y, t) \geq \alpha\right\}\right\| \leq 0 \\
& \Rightarrow\left\|\bigwedge\left\{t \succ \theta_{E}: N_{c}(x-y, t) \geq \alpha\right\}\right\|=0(\text { Since } 0<k<1) \\
& \Rightarrow \bigwedge\left\{t \succ \theta_{E}: N_{c}(x-y, t) \geq \alpha\right\}=\theta_{E} \\
& \Rightarrow \forall t \succ \theta_{E} ; N_{c}(x-y, t)>0 \\
& \Rightarrow x-y=\theta_{X} \text { by }(F C N 6) \\
& \Rightarrow x=y .
\end{aligned}
$$

Example 2 Let us consider the l-fuzzy complete cone normed linear space $\left(X, N_{c}, *\right)$ of Example 1. Let $T$ be self-map of $X$ given by $T x=\frac{x}{3}$. Take $k=\frac{1}{2}$.

$$
\begin{aligned}
& \text { Now, }\left\{t \succ \theta_{E}: N_{c}(T x-T y, t) \geq \alpha\right\}, \alpha \in(0,1) \\
& =\left\{t \succ \theta_{E}: N_{c}\left(\frac{x}{3}-\frac{y}{3}, t\right) \geq \alpha\right\} \\
& =\left\{t \succ \theta_{E}: \frac{\|x-y\|_{c}}{3} \prec t\right\} \\
& =\left\{t \succ \theta_{E}:\|x-y\|_{c} \prec 3 t\right\} \\
& \text { Again, }\left\{t \succ \theta_{E}: N_{c}\left(x-y, \frac{t}{k}\right) \geq \alpha\right\} \\
& =\left\{t \succ \theta_{E}: N_{c}(x-y, 2 t) \geq \alpha\right\} \\
& =\left\{t \succ \theta_{E}:\|x-y\|_{c} \prec 2 t\right\}
\end{aligned}
$$

Thus, $\bigwedge\left\{t \succ \theta_{E}:\|x-y\|_{c} \prec 3 t\right\} \preceq \bigwedge\left\{t \succ \theta_{E}:\|x-y\|_{c} \prec 2 t\right\}$

i.e., $\bigwedge\left\{t>\theta_{E}: N_{c}(T x-T y, t) \geq \alpha\right\} \preceq \bigwedge\left\{t>\theta_{E}: N_{c}\left(x-y, \frac{t}{k}\right) \geq \alpha\right\}$

Thus, $T$ satisfies Banach type contraction. We see that 0 is the unique fixed point of $T$.

Theorem 5 (Kannan contraction type fixed point theorem in fuzzy cone normed linear space) .

Let $\left(X, N_{c}, *\right)$ be a $l$-fuzzy complete cone normed linear space where $*=\min , P$ be a strongly minihedral cone with normal constant $M$. Suppose the mapping $T: X \longrightarrow X$ satisfies the contractive condition

$$
\begin{aligned}
& \bigwedge\left\{t \succ \theta_{E}: N_{c}(T x-T y, t) \geq \alpha\right\} \preceq k\left[\bigwedge\left\{t \succ \theta_{E}: N_{c}(T x-x, t) \geq \alpha\right\}\right. \\
& \left.\quad+\bigwedge\left\{t \succ \theta_{E}: N_{c}(T y-y, t) \geq \alpha\right\}\right]
\end{aligned}
$$

$\forall \alpha \in(0,1)$ and $k \in\left(0, \frac{1}{2}\right)$ is a constant. Then, $T$ has a unique fixed point in $X$.

Proof Choose $x_{0} \in X$. Set $x_{1}=T x_{0}, x_{2}=T x_{1}=T^{2} x_{0}, \ldots, x_{n+1}=T x_{n}=T^{n+1} x_{0}$. So $\left\{x_{n}\right\}$ is a sequence in $X$. First, we show that $\left\{x_{n}\right\}$ is $\alpha$-Cauchy sequence for all $\alpha \in(0,1)$. 
Now, for $\alpha \in$,

$$
\begin{aligned}
& \bigwedge\left\{t>\theta_{E}: N_{c}\left(x_{n+1}-x_{n}, t\right) \geq \alpha\right\} \\
& =\bigwedge\left\{t \succ \theta_{E}: N_{c}\left(T x_{n}-T x_{n-1}, t\right) \geq \alpha\right\} \\
& \leq k\left[\bigwedge\left\{t \succ \theta_{E}: N_{c}\left(T x_{n}-x_{n}, t\right) \geq \alpha\right\}+\bigwedge\left\{t \succ \theta_{E}: N_{c}\left(T x_{n-1}-x_{n-1}, t\right) \geq \alpha\right\}\right] \\
& =k\left[\bigwedge\left\{t \succ \theta_{E}: N_{c}\left(x_{n+1}-x_{n}, t\right) \geq \alpha\right\}+\bigwedge\left\{t \succ \theta_{E}: N_{c}\left(x_{n}-x_{n-1}, t\right) \geq \alpha\right\}\right] \\
& \Rightarrow \bigwedge\left\{t \succ \theta_{E}: N_{c}\left(x_{n+1}-x_{n}, t\right) \geq \alpha\right\} \preceq \frac{k}{1-k} \bigwedge\left\{t \succ \theta_{E}: N_{c}\left(x_{n}-x_{n-1}, t\right) \geq \alpha\right\} \\
& \Rightarrow \bigwedge\left\{t \succ \theta_{E}: N_{c}\left(x_{n+1}-x_{n}, t\right) \geq \alpha\right\} \preceq \delta^{n} \bigwedge\left\{t \succ \theta_{E}: N_{c}\left(x_{1}-x_{0}, t\right) \geq \alpha\right\} \\
& \text { where } \delta=\frac{k}{1-k}, 0<\delta<1 .
\end{aligned}
$$

Using normality condition, we get,

$$
\begin{aligned}
& \left\|\bigwedge\left\{t>\theta_{E}: N_{c}\left(x_{n+1}-x_{n}, t\right) \geq \alpha\right\}\right\| \leq M \delta^{n}\left\|\bigwedge\left\{t>\theta_{E}: N_{c}\left(x_{1}-x_{0}, t\right) \geq \alpha\right\}\right\| \\
& \Rightarrow \lim _{n \rightarrow \infty}\left\|\bigwedge\left\{t \succ \theta_{E}: N_{c}\left(x_{n+1}-x_{n}, t\right) \geq \alpha\right\}\right\|=0 . \\
& \Rightarrow \bigwedge\left\{t \succ \theta_{E}: N_{c}\left(x_{n+1}-x_{n}, t\right) \geq \alpha\right\} \rightarrow \theta_{E} \text { as } n \rightarrow \infty
\end{aligned}
$$

Now for $p \geq 1$ we have,

$$
\begin{aligned}
& \bigwedge\left\{t>\theta_{E}: N_{c}\left(x_{n+p}-x_{n+p-1}, \frac{t}{p}\right) \geq \alpha\right\} \\
& +\bigwedge\left\{t \succ \theta_{E}: N_{c}\left(x_{n+p-1}-x_{n+p-2}, \frac{t}{p}\right) \geq \alpha\right\} \\
& +\ldots+\bigwedge\left\{t \succ \theta_{E}: N_{c}\left(x_{n+1}-x_{n}, \frac{t}{p}\right) \geq \alpha\right\} \\
& \geq \bigwedge\left\{t>\theta_{E}: N_{c}\left(x_{n+p}-x_{n}, t\right) \geq \alpha * \alpha * \ldots * \alpha=\alpha\right\}
\end{aligned}
$$

Using normality condition and (4), it follows that

$$
\begin{aligned}
& \left\|\bigwedge\left\{t>\theta_{E}: N_{c}\left(x_{n+p}-x_{n}, t\right) \geq \alpha\right\}\right\| \rightarrow 0 \text { as } n \rightarrow \infty \text { for } p=1,2,3, \ldots \\
& \Rightarrow \bigwedge\left\{t \succ \theta_{E}: N_{c}\left(x_{n+p}-x_{n}, t\right) \geq \alpha\right\} \rightarrow \theta_{E} \text { as } n \rightarrow \infty \text { for } p=1,2,3, \ldots \\
& \Rightarrow\left\{x_{n}\right\} \text { is a } \alpha-\text { Cauchy sequence. }
\end{aligned}
$$

Since $X$ is $l$-fuzzy complete, thus $\exists x \in X$ such that

$$
\lim _{n \rightarrow \infty} \bigwedge\left\{t \succ \theta_{E}: N_{c}\left(x_{n}-x, t\right) \geq \alpha\right\}=\theta_{E}
$$

Now, 


$$
\begin{aligned}
& \bigwedge\left\{t>\theta_{E}: N_{c}(T x-x, t) \geq \alpha * \alpha\right\} \\
& \preceq \bigwedge\left\{t \succ \theta_{E}: N_{c}\left(T x-x_{n}, t\right) \geq \alpha\right\}+\bigwedge\left\{t>\theta_{E}: N_{c}\left(x_{n}-x, t\right) \geq \alpha\right\} \\
& =\bigwedge\left\{t \succ \theta_{E}: N_{c}\left(T x-T x_{n-1}, t\right) \geq \alpha\right\}+\bigwedge\left\{t \succ \theta_{E}: N_{c}\left(x_{n}-x, t\right) \geq \alpha\right\} \\
& \text { i.e., } \bigwedge\left\{t \succ \theta_{E}: N_{c}(T x-x, t) \geq \alpha\right\} \\
& \preceq k\left[\bigwedge\left\{t \succ \theta_{E}: N_{c}\left(T x_{n-1}-x_{n-1}, t\right) \geq \alpha\right\}+\bigwedge\left\{t \succ \theta_{E}: N_{c}(T x-x, t) \geq \alpha\right\}\right] \\
& +\bigwedge\left\{t \succ \theta_{E}: N_{c}\left(x_{n}-x, t\right) \geq \alpha\right\} \\
& \Rightarrow(1-k) \bigwedge\left\{t \succ \theta_{E}: N_{c}(T x-x, t) \geq \alpha\right\} \\
& \preceq k \bigwedge\left\{t \succ \theta_{E}: N_{c}\left(x_{n}-x_{n-1}, t\right) \geq \alpha\right\}+\bigwedge\left\{t \succ \theta_{E}: N_{c}\left(x_{n}-x, t\right) \geq \alpha\right\}
\end{aligned}
$$

Using normality condition, from (4) and (5), we get

$$
\begin{aligned}
& (1-k)\left\|\bigwedge\left\{t>\theta_{E}: N_{c}(T x-x, t) \geq \alpha\right\}\right\| \\
& \leq M\left\|k \bigwedge\left\{t>\theta_{E}: N_{c}\left(x_{n}-x_{n-1}, t\right) \geq \alpha\right\}+\bigwedge\left\{t \succ \theta_{E}: N_{c}\left(x_{n}-x, t\right) \geq \alpha\right\}\right\| \\
& \leq M k\left\|\bigwedge\left\{t>\theta_{E}: N_{c}\left(x_{n}-x_{n-1}, t\right) \geq \alpha\right\}\right\|+M\left\|\bigwedge\left\{t \succ \theta_{E}: N_{c}\left(x_{n}-x, t\right) \geq \alpha\right\}\right\| \\
& \Rightarrow\left\|\bigwedge\left\{t \succ \theta_{E}: N_{c}(T x-x, t) \geq \alpha\right\}\right\|=0 \text { as } n \rightarrow \infty \\
& \Rightarrow \bigwedge\left\{t>\theta_{E}: N_{c}(T x-x, t) \geq \alpha\right\}=\theta_{E} \\
& \Rightarrow N_{c}(T x-x, t) \geq \alpha \forall \alpha \in(0,1) \text { and } \forall t \succ \theta_{E} \\
& \Rightarrow N_{c}(T x-x, t)=1 \forall t \succ \theta_{E} \\
& \Rightarrow T x-x=\theta_{X} \text { by }(F C N 2) \\
& \Rightarrow T x=x .
\end{aligned}
$$

Thus, $T$ has a fixed point.

Uniqueness: If $\exists y \in X$ such that $T y=y$.

Now,

$$
\begin{aligned}
& \bigwedge\left\{t>\theta_{E}: N_{c}(x-y, t) \geq \alpha\right\} \\
& =\bigwedge\left\{t \succ \theta_{E}: N_{c}(T x-T y, t) \geq \alpha\right\} \\
& \preceq k\left[\bigwedge\left\{t \succ \theta_{E}: N_{c}(T x-x, t) \geq \alpha\right\}+\bigwedge\left\{t \succ \theta_{E}: N_{c}(T y-y, t) \geq \alpha\right\}\right]
\end{aligned}
$$

Using normality condition, we have

$$
\begin{aligned}
& \left\|\bigwedge\left\{t>\theta_{E}: N_{c}(x-y, t) \geq \alpha\right\}\right\| \\
& \leq M k\left\|\bigwedge\left\{t>\theta_{E}: N_{c}(T x-x, t) \geq \alpha\right\}\right\|+M k\left\|\bigwedge\left\{t \succ \theta_{E}: N_{c}(T y-y, t) \geq \alpha\right\}\right\| \\
& \Rightarrow\left\|\bigwedge\left\{t \succ \theta_{E}: N_{c}(x-y, t) \geq \alpha\right\}\right\|=0 . \\
& \Rightarrow \bigwedge\left\{t>\theta_{E}: N_{c}(x-y, t) \geq \alpha\right\}=\theta_{E} . \\
& \Rightarrow N_{c}(x-y, t) \geq \alpha \forall \alpha \in(0,1) \text { and } \forall t \succ \theta_{E} \\
& \Rightarrow N_{c}(x-y, t)=1 \forall t \succ \theta_{E} \\
& \Rightarrow x-y=\theta_{X} \text { by }(F C N 2) \\
& \Rightarrow x=y .
\end{aligned}
$$


Example 3 Let us consider the l-fuzzy complete cone normed linear space $\left(X, N_{c}, *\right)$ of Example 1. Let $T$ be self-map of X given by $T x=\frac{x}{6}$. Take $k=\frac{1}{5}$.

Now,

$$
\begin{aligned}
& \bigwedge\left\{t \succ \theta_{E}: N_{c}\left(T x-x, \frac{t}{k}\right) \geq \alpha\right\}+\bigwedge\left\{t \succ \theta_{E}: N_{c}\left(T y-y, \frac{t}{k}\right) \geq \alpha\right\} \\
& \geq \bigwedge\left\{s+t \succ \theta_{E}: N_{c}\left(T x-x+y-T y, \frac{s+t}{k}\right) \geq \alpha\right\} \\
& =\bigwedge\left\{s+t \succ \theta_{E}: N_{c}\left(\frac{5 y}{6}-\frac{5 x}{6}, \frac{s+t}{\frac{1}{5}}\right) \geq \alpha\right\} \\
& =\bigwedge\left\{s+t \succ \theta_{E}: N_{c}\left(\frac{x}{6}-\frac{y}{6}, s+t\right) \geq \alpha\right\} \\
& =\bigwedge\left\{t \succ \theta_{E}: N_{c}\left(\frac{x}{6}-\frac{y}{6}, t\right) \geq \alpha\right\} \\
& =\bigwedge\left\{t \succ \theta_{E}: N_{c}(T x-T y, t) \geq \alpha\right\}
\end{aligned}
$$

Thus, $T$ satisfies Kannan type contraction. We see that 0 is the unique fixed point of $T$.

Theorem 6 (Chatterjee contraction type fixed point theorem.)

Let $\left(X, N_{c}, *\right)$ be a $l$-fuzzy complete cone normed linear space where $*=\min , P$ be a stronghly minihedral cone with normal constant $M$. Suppose the mapping $T: X \longrightarrow X$ satisfies the contractive condition

$$
\begin{aligned}
\bigwedge\{t & \left.>\theta_{E}: N_{c}(T x-T y, t) \geq \alpha\right\} \\
& \leq k\left[\bigwedge\left\{t \succ \theta_{E}: N_{c}(T x-y, t) \geq \alpha\right\}+\bigwedge\left\{t \succ \theta_{E}: N_{c}(T y-x, t) \geq \alpha\right\}\right]
\end{aligned}
$$

$\forall \alpha \in(0,1)$ and $k \in\left(0, \frac{1}{2}\right)$ is a constant. Then $T$ has a fixed point in $X$. In addition, if $M=1$, then the fixed point is unique.

Proof Choose $x_{0} \in X$. Set $x_{1}=T x_{0}, x_{2}=T x_{1}=T^{2} x_{0}, \ldots, x_{n+1}=T x_{n}=T^{n+1} x_{0}$. So $\left\{x_{n}\right\}$ is a sequence in $X$. First, we show that $\left\{x_{n}\right\}$ is $\alpha$-Cauchy sequence for all $\alpha \in(0,1)$.

Now for $\alpha \in(0,1)$;

$$
\begin{aligned}
& \bigwedge\left\{t \succ \theta_{E}: N_{c}\left(x_{n+1}-x_{n}, t\right) \geq \alpha\right\} \\
& =\bigwedge\left\{t \succ \theta_{E}: N_{c}\left(T x_{n}-T x_{n-1}, t\right) \geq \alpha\right\} \\
& \preceq k\left[\bigwedge\left\{t>\theta_{E}: N_{c}\left(T x_{n}-x_{n-1}, t\right) \geq \alpha\right\}+\bigwedge\left\{t>\theta_{E}: N_{c}\left(T x_{n-1}-x_{n}, t\right) \geq \alpha\right\}\right] \\
& =k\left[\bigwedge\left\{t \succ \theta_{E}: N_{c}\left(x_{n+1}-x_{n-1}, t\right) \geq \alpha\right\}+\bigwedge\left\{t \succ \theta_{E}: N_{c}\left(x_{n}-x_{n}, t\right) \geq \alpha\right\}\right] \\
& \preceq k\left[\bigwedge\left\{t \succ \theta_{E}: N_{c}\left(x_{n+1}-x_{n}, t\right) \geq \alpha\right\}+\bigwedge\left\{t \succ \theta_{E}: N_{c}\left(x_{n}-x_{n-1}, t\right) \geq \alpha\right\}\right]+\theta_{E} \\
& \Rightarrow \bigwedge\left\{t \succ \theta_{E}: N_{c}\left(x_{n+1}-x_{n}, t\right) \geq \alpha\right\} \preceq \frac{k}{1-k} \bigwedge\left\{t \succ \theta_{E}: N_{c}\left(x_{n}-x_{n-1}, t\right) \geq \alpha\right\} \\
& \Rightarrow \bigwedge\left\{t \succ \theta_{E}: N_{c}\left(x_{n+1}-x_{n}, t\right) \geq \alpha\right\} \preceq \delta^{n} \bigwedge\left\{t \succ \theta_{E}: N_{c}\left(x_{1}-x_{0}, t\right) \geq \alpha\right\} \text { where } \\
& \delta=\frac{k}{1-k}, 0<\delta<1 .
\end{aligned}
$$

Using normality condition, we get 


$$
\begin{aligned}
& \left\|\bigwedge\left\{t \succ \theta_{E}: N_{c}\left(x_{n+1}-x_{n}, t\right) \geq \alpha\right\}\right\| \leq M \delta^{n}\left\|\bigwedge\left\{t \succ \theta_{E}: N_{c}\left(x_{1}-x_{0}, t\right) \geq \alpha\right\}\right\| \\
& \Rightarrow \lim _{n \rightarrow \infty}\left\|\bigwedge\left\{t \succ \theta_{E}: N_{c}\left(x_{n+1}-x_{n}, t\right) \geq \alpha\right\}\right\|=0 . \\
& \Rightarrow \lim _{n \rightarrow \infty} \bigwedge\left\{t \succ \theta_{E}: N_{c}\left(x_{n+1}-x_{n}, t\right) \geq \alpha\right\}=\theta_{E}
\end{aligned}
$$

Now for $p \geq 1$, we have

$$
\begin{aligned}
& \bigwedge\left\{t>\theta_{E}: N_{c}\left(x_{n+p}-x_{n+p-1}, \frac{t}{p}\right) \geq \alpha\right\}+\bigwedge\left\{t \succ \theta_{E}: N_{c}\left(x_{n+p-1}-x_{n+p-2}, \frac{t}{p}\right) \geq \alpha\right\} \\
& +\ldots+\bigwedge\left\{t \succ \theta_{E}: N_{c}\left(x_{n+1}-x_{n}, \frac{t}{p}\right) \geq \alpha\right\} \succeq \bigwedge\left\{t>\theta_{E}: N_{c}\left(x_{n+p}-x_{n}, t\right) \geq \alpha * \alpha * \ldots * \alpha=\alpha\right\}
\end{aligned}
$$

Using normality condition and (6), it follows that

$$
\begin{aligned}
& \left\|\bigwedge\left\{t>\theta_{E}: N_{c}\left(x_{n+p}-x_{n}, t\right) \geq \alpha\right\}\right\| \rightarrow 0 \text { as } n \rightarrow \infty \text { for } p=1,2,3, \ldots \\
& \Rightarrow \bigwedge\left\{t>\theta_{E}: N_{c}\left(x_{n+p}-x_{n}, t\right) \geq \alpha\right\} \rightarrow \theta_{E} \text { as } n \rightarrow \infty \text { for } p=1,2,3, \ldots \\
& \Rightarrow\left\{x_{n}\right\} \text { is a } \alpha \text { - Cauchy sequence. }
\end{aligned}
$$

Since $X$ is $l$-fuzzy complete, thus $\exists x \in X$ such that

$$
\lim _{n \rightarrow \infty} \bigwedge\left\{t \succ \theta_{E}: N_{c}\left(x_{n}-x, t\right) \geq \alpha\right\}=\theta_{E}
$$

Now,

$$
\begin{aligned}
& \bigwedge\left\{t \succ \theta_{E}: N_{c}(T x-x, t) \geq \alpha * \alpha\right\} \\
& \preceq \bigwedge\left\{t \succ \theta_{E}: N_{c}\left(T x-x_{n}, t\right) \geq \alpha\right\}+\bigwedge\left\{t \succ \theta_{E}: N_{c}\left(x_{n}-x, t\right) \geq \alpha\right\} \\
& =\bigwedge\left\{t \succ \theta_{E}: N_{c}\left(T x-T x_{n-1}, t\right) \geq \alpha\right\}+\bigwedge\left\{t \succ \theta_{E}: N_{c}\left(x_{n}-x, t\right) \geq \alpha\right\} \\
& \preceq k\left[\bigwedge\left\{t \succ \theta_{E}: N_{c}\left(T x-x_{n-1}, t\right) \geq \alpha\right\}+\bigwedge\left\{t \succ \theta_{E}: N_{c}\left(T x_{n-1}-x, t\right) \geq \alpha\right\}\right] \\
& +\bigwedge\left\{t \succ \theta_{E}: N_{c}\left(x_{n}-x, t\right) \geq \alpha\right\} \\
& =k \bigwedge\left\{t \succ \theta_{E}: N_{c}\left(T x-x_{n-1}, t\right) \geq \alpha\right\}+(1+k) \bigwedge\left\{t \succ \theta_{E}: N_{c}\left(x_{n}-x, t\right) \geq \alpha\right\} \\
& \preceq k\left[\bigwedge\left\{t \succ \theta_{E}: N_{c}(T x-x, t) \geq \alpha\right\}+\bigwedge\left\{t \succ \theta_{E}: N_{c}\left(x_{n-1}-x, t\right) \geq \alpha\right\}\right] \\
& +(1+k) \bigwedge\left\{t \succ \theta_{E}: N_{c}\left(x_{n}-x, t\right) \geq \alpha\right\} \\
& \Rightarrow(1-k) \bigwedge\left\{t \succ \theta_{E}: N_{c}(T x-x, t) \geq \alpha\right\} \preceq k \bigwedge\left\{t \succ \theta_{E}: N_{c}\left(x_{n-1}-x, t\right) \geq \alpha\right\} \\
& +(1+k) \bigwedge\left\{t \succ \theta_{E}: N_{c}\left(x_{n}-x, t\right) \geq \alpha\right\}
\end{aligned}
$$

Using normality condition and (8), we get 


$$
\begin{aligned}
& \left\|(1-k) \bigwedge\left\{t \succ \theta_{E}: N_{c}(T x-x, t) \geq \alpha\right\}\right\| \\
& \left.\leq M \| k \bigwedge\left\{t \succ \theta_{E}: N_{c}\left(x_{n-1}-x, t\right) \geq \alpha\right\}\right]+(1+k) \bigwedge\left\{t \succ \theta_{E}: N_{c}\left(x_{n}-x, t\right) \geq \alpha\right\} \| \\
& \Rightarrow\left\|\bigwedge\left\{t \succ \theta_{E}: N_{c}(T x-x, t) \geq \alpha\right\}\right\| \leq \frac{M k}{1-k}\left\|\bigwedge\left\{t \succ \theta_{E}: N_{c}\left(x_{n-1}-x, t\right) \geq \alpha\right\}\right\| \\
& +\frac{M(1+k)}{1-k}\left\|\bigwedge\left\{t \succ \theta_{E}: N_{c}\left(x_{n}-x, t\right) \geq \alpha\right\}\right\| \\
& \Rightarrow\left\|\bigwedge\left\{t \succ \theta_{E}: N_{c}(T x-x, t) \geq \alpha\right\}\right\|=0 \text { as } n \rightarrow \infty \\
& \Rightarrow \bigwedge\left\{t \succ \theta_{E}: N_{c}(T x-x, t) \geq \alpha\right\}=\theta_{E} \\
& \Rightarrow N_{c}(T x-x, t) \geq \alpha \forall \alpha \in(0,1) \text { and } \forall t \succ \theta_{E} \\
& \Rightarrow N_{c}(T x-x, t)=1 \forall t \succ \theta_{E} \\
& \Rightarrow T x-x=\theta_{X} \text { by }(F C N 2) \\
& \Rightarrow T x=x .
\end{aligned}
$$

Thus, $T$ has a fixed point.

Uniqueness: If $\exists y \in X$ such that $T y=y$.

Now,

$$
\begin{aligned}
& \bigwedge\left\{t \succ \theta_{E}: N_{c}(x-y, t) \geq \alpha\right\} \\
& =\bigwedge\left\{t \succ \theta_{E}: N_{c}(T x-T y, t) \geq \alpha\right\} \\
& \preceq k\left[\bigwedge\left\{t \succ \theta_{E}: N_{c}(T x-y, t) \geq \alpha\right\}+\bigwedge\left\{t \succ \theta_{E}: N_{c}(T y-x, t) \geq \alpha\right\}\right] \\
& =k\left[\bigwedge\left\{t \succ \theta_{E}: N_{c}(x-y, t) \geq \alpha\right\}+\bigwedge\left\{t \succ \theta_{E}: N_{c}(y-x, t) \geq \alpha\right\}\right] \\
& =2 k \bigwedge\left\{t \succ \theta_{E}: N_{c}(x-y, t) \geq \alpha\right\}
\end{aligned}
$$

Using normality condition with normal constant 1 , we get

$$
\begin{aligned}
& \left\|\bigwedge\left\{t \succ \theta_{E}: N_{c}(x-y, t) \geq \alpha\right\}\right\| \leq\left\|2 k \bigwedge\left\{t \succ \theta_{E}: N_{c}(x-y, t) \geq \alpha\right\}\right\| \\
& \Rightarrow(1-2 k)\left\|\bigwedge\left\{t \succ \theta_{E}: N_{c}(x-y, t) \geq \alpha\right\}\right\| \leq 0 \\
& \Rightarrow\left\|\bigwedge\left\{t \succ \theta_{E}: N_{c}(x-y, t) \geq \alpha\right\}\right\|=0 .\left(\text { Since } 0<k<\frac{1}{2}\right) \\
& \Rightarrow \bigwedge\left\{t \succ \theta_{E}: N_{c}(x-y, t) \geq \alpha\right\}=\theta_{E} . \\
& \Rightarrow N_{c}(x-y, t) \geq \alpha \forall \alpha \in(0,1) \text { and } \forall t \succ \theta_{E} \\
& \Rightarrow N_{c}(x-y, t)=1 \forall t \succ \theta_{E} \\
& \Rightarrow x-y=\theta_{X} \text { by }(F C N 2) \\
& \Rightarrow x=y .
\end{aligned}
$$

Example 4 Let us consider the l-fuzzy complete cone normed linear space $\left(X, N_{c}, *\right)$ of Example 1. Let $T$ be self-map of $X$ given by $T x=\frac{x}{2}$. Take $k=\frac{1}{3}$. 
Now,

$$
\begin{aligned}
& \bigwedge\left\{t \succ \theta_{E}: N_{c}\left(T x-y, \frac{t}{k}\right) \geq \alpha\right\}+\bigwedge\left\{t \succ \theta_{E}: N_{c}\left(T y-x, \frac{t}{k}\right) \geq \alpha\right\} \\
& \succeq \bigwedge\left\{s+t \succ \theta_{E}: N_{c}\left(T x-y+x-T y, \frac{s+t}{k}\right) \geq \alpha\right\} \\
& =\bigwedge\left\{s+t \succ \theta_{E}: N_{c}\left(\frac{3 x}{2}-\frac{3 y}{2}, \frac{s+t}{\frac{1}{3}}\right) \geq \alpha\right\} \\
& =\bigwedge\left\{s+t \succ \theta_{E}: N_{c}\left(\frac{x}{2}-\frac{y}{2}, s+t\right) \geq \alpha\right\} \\
& =\bigwedge\left\{t \succ \theta_{E}: N_{c}\left(\frac{x}{2}-\frac{y}{2}, t\right) \geq \alpha\right\} \\
& =\bigwedge\left\{t \succ \theta_{E}: N_{c}(T x-T y, t) \geq \alpha\right\}
\end{aligned}
$$

Thus, $T$ satisfies Chatterjee type contraction. We see that 0 is the unique fixed point of $T$.

\section{Acknowledgements}

The authors are grateful to the editor and reviewers for their valuable comments.

\section{Authors' contributions}

Both authors jointly worked on the results and approved the final manuscript.

\section{Funding}

Not applicable

\section{Availability of data and materials}

Not applicable

\section{Competing interests}

The authors declare that they have no competing interests.

\section{Author details \\ ${ }^{1}$ Department of Mathematics, Surya Sen Mahavidyalaya, Siliguri 734004, India. ${ }^{2}$ Tarapada Bag, Department of} Mathematics, Visva-Bharati, Santiniketan 731235, India.

Received: 6 June 2019 Accepted: 9 October 2019

Published online: 19 November 2019

\section{References}

1. Katsaras, A. K.: Fuzzy topological vector spaces. Fuzzy Sets Syst. 12(1984), 143-154

2. Felbin, C.: Finite Dimensional Fuzzy Normed Linear Spaces. Fuzzy Sets Syst. 48, 239-248 (1992)

3. Kaleva, O., Seikkala, S.: On fuzzy metric spaces. Fuzzy Sets Syst. 12, 215-229 (1984)

4. Cheng, S. C., Mordeson, J. N.: Fuzzy linear operators and fuzzy normed linear spaces. Bull. Calcutta. Math. Soc. 86(5), 429-436 (1994)

5. Kramosil, I., Michalek, J.: Fuzzy metric and statistical metric spaces. Kybernetica. 11, 326-334 (1975)

6. Bag, T., Samanta, S. K.: Finite dimensional fuzzy normed linear spaces. J. Fuzzy Math. 11(3), 687-705 (2003)

7. Long-Guang, H., Xian, Z.: Cone metric spaces and fixed point theorems of contractive mappings. J. Math. Anal. Appl. $332,1468-1476(2007)$

8. Tamang, P, Bag, T: Some results on finite dimensional fuzzy cone normed linear space. Ann. Fuzzy Math. Inform. 13(1), 123-134 (2017)

9. Banach, S.: Sur les operations dans les ensembles obstraits et. Leur. Application aux equation integrales. Fund. Math. 3, 133-181 (1922)

10. Kannan, R.: Some results on fixed point. Bull. Cal. Math. Soc. 60, 71-76 (1968)

11. Chatterjee, S. K.: Fixed point theorem, C. R. Acad. Bulg. Sci. 25, 727-30 (1972)

12. Deimling, K.: Nonlinear Functional Analysis. Springer, Berlin (1985)

13. Klir, G. J., Yuan, B.: Fuzzy sets and fuzzy logic: theory and applications, p. 563. Upper Saddle River, (1995)

\section{Publisher's Note}

Springer Nature remains neutral with regard to jurisdictional claims in published maps and institutional affiliations. 